SLAC-PUB-8791

BABAR-PROC-01/08

March 27th, 2001

\title{
B Mixing and Lifetime Measurements with the BABAR Detector.
}

\author{
Concezio Bozzi \\ INFN Sezione di Ferrara \\ Via Paradiso 12, I-44100 Ferrara, Italy \\ (representing the BABAR Collaboration)
}

\begin{abstract}
Recent $B A B A R$ measurements on lifetime and mixing of $B$ mesons are reported. Various techniques are used, ranging from the full reconstruction of hadronic $B$ decays, to partial reconstruction techniques, and to a totally inclusive approach with dilepton events. The results presented are based on a data sample collected by BABAR during the 1999-2000 data taking, and should be considered as preliminary.
\end{abstract}

Contributed to the Proceedings of the $4^{\text {th }}$ International Workshop on B Physics and CP Violation (BCP4), 2/19/2001-2/23/2001, Ise, Japan 


\section{Introduction}

A precision measurement of the $B^{0} \bar{B}^{0}$ oscillation frequency is of great importance since this quantity is sensitive to the CKM matrix element $\left|V_{t d}\right|$ and, in combination with knowledge of the $B_{s}^{0} \bar{B}_{s}^{0}$ oscillation frequency, provides a stringent constraint on the Unitarity Triangle. A precision measurement of $B$ meson lifetimes is also of great importance for the understanding of the dynamics involved in heavy quark decays. Moreover, in the specific case of the new asymmetric B-Factories, both lifetime and time-dependent mixing measurements are powerful tools for understanding the performance of both the detector and the analysis algorithms, and therefore they represent validation analyses for the measurements of time-dependent CP asymmetries.

This paper presents a set of $B$ meson lifetimes and mixing measurements performed with the BABAR detector [1] at the PEP-II $e^{+} e^{-}$asymmetric B-Factory. Several techniques are used, ranging from fully exclusive to completely inclusive event selections. Exclusive measurements generally offer smaller systematic uncertainties with respect to inclusive ones, but they also suffer from smaller branching fractions, and consequently larger statistical uncertainties. The following sections will detail the three main mixing and lifetime measurement procedures followed in BABAR namely the selection of events

- where one $B$ meson decay is fully reconstructed in a hadronic flavour eigenstate, or

- containing two high momentum leptons from semileptonic $B$ meson decays (dilepton events), or

- where a $B$ decay is reconstructed by using a semi-exclusive technique in either an hadronic or a $D^{*} \ell \nu$ final state.

The data sample used was collected by the BABAR detector at the PEP-II asymmetric B-Factory, during the years 1999-2000. Unless otherwise specified, the integrated luminosities are $20.7 \mathrm{fb}^{-1}$ on the $\Upsilon(4 \mathrm{~S})$ peak, and $2.6 \mathrm{fb}^{-1} 40 \mathrm{MeV}$ below resonance. All results are preliminary.

\section{Mixing and Lifetimes with fully reconstructed hadronic $B$ de- cays}

This technique is the most similar to the measurement of the time-dependent CP asymmetry also presented at this Conference [2, 3], the only difference being the exclusive reconstruction of a flavour eigenstate instead of a $\mathrm{CP}$ eigenstate. The main ingredients necessary to perform these time-dependent measurements are:

1. to exclusively reconstruct a flavour-eigenstate hadronic final state $\left(B_{\text {reco }}\right)$,

2. to tag the flavour of the other $B$ meson $\left(B_{t a g}\right)$ 円,

3. to measure the time difference $\Delta t$ between the two $B$ meson decays.

Since $B$ production at the $\Upsilon(4 \mathrm{~S})$ resonance is coherent, tagging the flavour of a $B$ meson at its decay time will unambigously determine the flavour of the other $B$ at the same time. The distribution of

\footnotetext{
${ }^{1}$ This step is not needed for lifetime measurements.
} 
the time difference $\Delta t$ between $B$ meson decays with the opposite $\left(h_{+}\right)$or the same $\left(h_{-}\right)$flavour is therefore

$$
h_{ \pm}=1 / 4 \Gamma e^{-\Gamma|\Delta t|}\left(1 \pm(1-2 w) \cos \left(\Delta m_{d} \Delta t\right)\right)
$$

where $\Gamma=1 / \tau_{B^{0}}$ and $w$ is the probability to get a wrong flavour tagging. The resulting same/opposite flavour asymmetry will have a pure cosine time dependence. Equation 1 has to be modified to take into account the detector resolution function and backgrounds.

\subsection{Exclusive reconstruction of hadronic $B$ decays}

Several hadronic decays are reconstructed], based on most favourable production rates and low background contamination:

$$
\begin{array}{rlll}
\bar{B}^{0} \rightarrow & D^{(*)+} \pi^{-} & B^{-} \rightarrow & D^{(*) 0} \pi^{-} \\
& D^{(*)+} \rho^{-} & & J / \psi K^{-} \\
& D^{(*)+} a_{1}^{-} & & \psi(2 S) K^{-} \\
& J / \psi \bar{K}^{* 0} & &
\end{array}
$$

where $D^{*+} \rightarrow D^{0} \pi^{+}, D^{* 0} \rightarrow D^{0} \pi^{0}, \psi(2 S) \rightarrow J / \psi \pi^{+} \pi^{-}$or $\ell^{+} \ell^{-}, J / \psi \rightarrow \ell^{+} \ell^{-}, \bar{K}^{* 0} \rightarrow K^{-} \pi^{+}$. Neutral $D$ mesons are reconstructed in the $K^{-} \pi^{+}, K^{-} \pi^{+} \pi^{-} \pi^{+}, K^{-} \pi^{+} \pi^{0}$ and (except for $B^{-} \rightarrow D^{* 0} \pi^{-}$) $K_{s}^{0} \pi^{+} \pi^{-}$modes, charged $D$ mesons in the $K^{-} \pi^{+} \pi^{+}$and $K_{s}^{0} \pi^{+}$modes. Signal to background discrimination is based on two variables, the beam energy substituted mass, $m_{E S}$, and the energy difference $\Delta E$, defined as

$$
m_{E S}=\sqrt{E_{\text {beam }}^{* 2}-p_{B}^{* 2}} \quad \text { and } \quad \Delta E=E_{B}^{*}-E_{\text {beam }}^{*},
$$

where $E_{\text {beam }}^{*}, p_{B}^{*}$ and $E_{B}^{*}$ are respectively the beam energy and the momentum and energy of the fully reconstructed $B$ meson, computed in the center-of-mass (CMS) frame. The $m_{E S}$ resolution is about $2.6 \mathrm{MeV}$ and is dominated by the beam energy spread, whereas the resolution on $\Delta E$ is mode-dependent and varies between 20 and $30 \mathrm{MeV}$, being worse in decay chains involving neutral pions. The $m_{E S}$ distribution for neutral $B$ decays, after applying a $3 \sigma$ cut on $\Delta E$, is shown in Figure 1. The number of neutral (charged) B signal events with $m_{E S}>5.27 \mathrm{GeV} / c^{2}$ is $6643 \pm 96$ $(6928 \pm 94)$, with a purity of about $84 \%(87 \%)$. The combinatorial background is estimated by fitting the $m_{E S}$ distribution between 5.2 and $5.3 \mathrm{GeV} / c^{2}$ with the sum of a gaussian for signal and an Argus function [4] for background. This procedure also allows to assign a signal probability on an event-by-event basis, which is used in the fitting procedure. The time dependence of combinatorial background is determined from the time distribution of events in the sideband region. In addition to combinatorial background, there is a small contribution which peaks in the signal region. This peaking background is due to pion/photon swapping between the two $B$ mesons in the event, and introduces a contamination from charged $B$ mesons in neutral $B$ decays and vice-versa. Detailed Monte-Carlo studies show that peaking background is at the percent level.

\subsection{B-flavour tagging and particle identification}

After reconstructing a full $B$ meson decay chain $\left(B_{\text {reco }}\right)$, the remaining tracks of the event are analyzed to determine the $B_{t a g}$ flavour. Powerful tagging signatures are primary leptons in semileptonic

\footnotetext{
${ }^{2}$ charge conjugation is always implied in the following.
} 


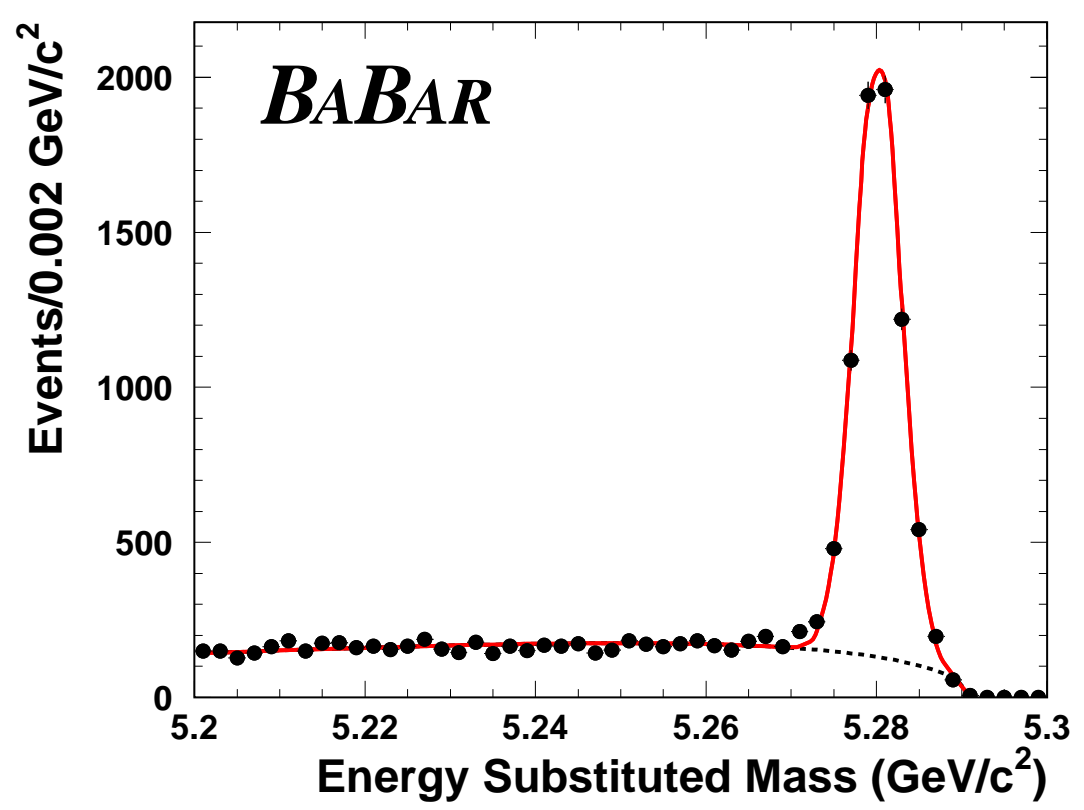

Figure 1: Beam energy substituted mass distribution for fully reconstructed hadronic neutral $B$ decays.

$B$ decays, kaons resulting from $b \rightarrow c \rightarrow s$ transitions and slow pions from $D^{*}$ in $B \rightarrow D^{*} X$ decays. The tagging algorithm assigns events to one (and only one) of the following categories, in order of decreasing priority:

- primary lepton tag (electron with $p>1 \mathrm{GeV} / c$ in the CMS or muon with $p>1.1 \mathrm{GeV} / c$ in the CMS if no electron is found), with the tag determined by the lepton charge;

- kaon tag, with tagging defined by the sum of all kaon charges (required to be non-zero);

- NT1 and NT2 tags, based on the output of a neural network.

NT1 and NT2 are aimed at recovering leptons which do not pass the requirements for the primary lepton tag, resolving ambiguous cases where a lepton tag has a conflicting kaon tag, and identifying slow pions from $D^{*}$ decays. Figure 2 shows the tagging efficiency $\varepsilon$ versus the fraction of wrong tags $w$. The figure of merit for tagging is defined as $Q=\varepsilon(1-2 w)^{2}$. The kaon tagging category has the highest $Q$, followed by lepton, NT1 and NT2 tags. The number of tagged neutral $B$ events is $4538 \pm 75$, which gives a tagging efficiency of $68 \%$. The mistag fractions are determined from the fit to data in the mixing analysis (see 2.4).

Most of the tagging power relies on particle identification [1], which has therefore to be optimized for maximal efficiency and low misidentification probability.

Electron identification is based on track matching with a calorimeter cluster, on the momentumto-energy ratio $(0.89<E / p<1.2$, where $E$ and $p$ are the energy measured in the calorimeter and the track momentum measured in the drift chamber, respectively), on requirements on the electromagnetic shower shape, and on consistency for $d E / d x$ and the Cherenkov angle measured in the DIRC (when available) with the electron hypothesis. 


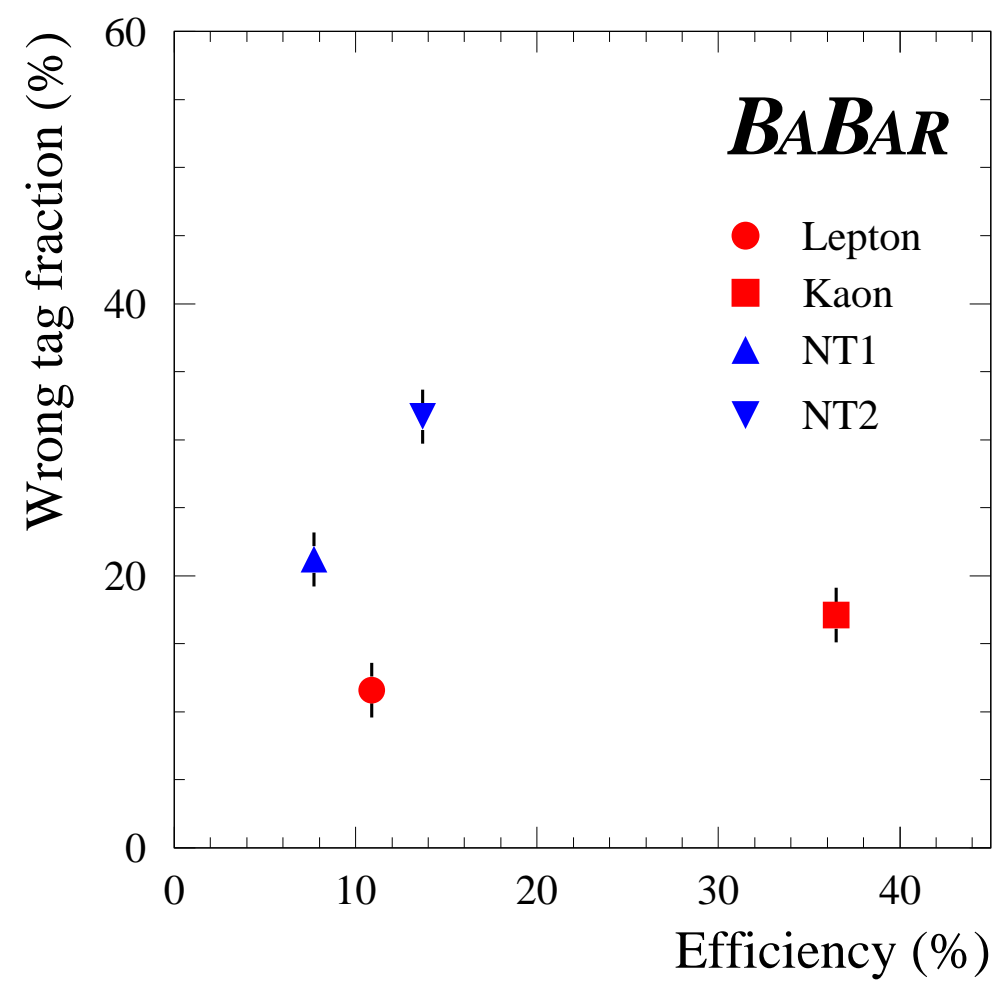

Figure 2: Tagging efficiencies vs. wrong tag probability for the four tagging categories described in the text.

Muon identification relies mainly on the Instrumented Flux Return (IFR), namely on the number of measured interaction lengths (greater than 2), on the difference between the measured and the expected number of interaction lenghts (less than 1), on matching between IFR hits and the extrapolated track, and on requirements on the average number and spread of IFR hits per layer.

Kaons are identified with a neural network based on likelihood ratios computed from quantities measured in the DCH and SVT $(d E / d x)$, and in DIRC, where single hits are compared with the pattern expected for Cherenkov light in the kaon/pion hypotheses. Kaon/pion separation is above $3 \sigma$ for momenta up to $3.5 \mathrm{GeV} / c$.

Table 1 shows the efficiencies and pion misidentification rates for electrons, muons and kaons, as determined from data control samples.

\subsection{Vertexing and time measurement}

The time difference between the two $B$ meson decays $\Delta t$ is inferred from the distance between their decay vertices along the beam line, $\Delta z$, via the boost factor:

$$
\Delta t=\Delta z /(\beta \gamma c)
$$

Since $\langle\beta \gamma\rangle=0.56$ at PEP-II, the average separation between the two $B$ decay vertices is about $250 \mu \mathrm{m}$, which is measurable by a vertex detector. The $B_{\text {reco }}$ decay vertex is conceptually easily identified, whereas the $B_{\text {tag }}$ vertex has to be reconstructed inclusively by using all other tracks 
Table 1: Efficiencies and pion misidentification probabilities for electrons, muons and kaons.

\begin{tabular}{|c|c|c|}
\hline Particle & Efficiency (\%) & Pion misid. (\%) \\
\hline Electrons & 91 & 0.13 \\
Muons & 75 & 2.5 \\
Kaons & 85 & 5 \\
\hline
\end{tabular}

Table 2: Resolution function parameters for the $z$ position of the fully reconstructed vertex and for $\Delta z$.

\begin{tabular}{|c|c|c|}
\hline Parameter & $z_{\text {Breco }}$ & $\Delta z$ \\
\hline Core $\sigma(\mu \mathrm{m})$ & 45 & 100 \\
Core fraction $(\%)$ & 80 & 70 \\
RMS $(\mu \mathrm{m})$ & 70 & 170 \\
\hline
\end{tabular}

in the event. Particular care has to be taken in order to avoid any bias coming from tracks not originating from the tagging $B$ vertex:

- neutral long-lived particle $\left(\right.$ e.g. $\left.K^{0}, \Lambda\right)$ are searched for and used in the vertex fit instead of their charged decay products;

- tracks originating from secondary (charm) decay vertices are excluded from the vertex fit by an algorithm which removes iteratively the track which gives the biggest chi-square difference between the vertices reconstructed with and without the track itself. The procedure is repeated until the chi-square difference for every remaining track is less than 6 or until there are no remaining tracks.

Additional kinematic constraints derived from the $B_{\text {reco }}$ momentum and decay vertex position, from the beam spot position and size and from the knowledge of the boost, are used during the reconstruction of the $B_{t a g}$ vertex.

The resulting $\Delta z$ resolution function is parametrized with three gaussians (core, tail, outlier) for the mixing fit, and with a sum of a gaussian and the same gaussian convoluted with an exponential decay for the lifetime fit. Table 2 reports the resolution parameters for the $z$ position of the fully reconstructed vertex and for $\Delta z$. The dominant contribution to the $\Delta z$ resolution is due to the tagging vertex. In the mixing fit, the widths of the core and tail gaussians are scaled on an eventby-event basis with the error computed from the vertex fits, whereas the outlier gaussian has a fixed width of $8 p s$. A possible remaining bias due to secondary vertex tracks not removed in the fitting procedure is taken into account by allowing a non-zero mean of the core and tail gaussians. Since the bias depends on tagging category, different core biases are allowed for each of them. Most of the resolution function parameters are fitted in data: two scale factors for the core and tail widths, one tail and four core biases, the relative core/tail/outlier amounts, and a bias for the outliers. 


\subsection{Results}

An unbinned maximum likelihood technique is applied to fit simultaneously the distribution of mixed and unmixed events. The signal is parametrized as in Equation 1, whereas the combinatorial background is taken into account by a zero-lifetime and a non-oscillatory, non-zero lifetime components, both convoluted with a two-gaussian background resolution function. Fit parameters are 34: $\Delta m_{d}$, the mistag fractions $w$ for the four tagging categories, the signal and background resolution functions, and the background parameters. The correlation between any other parameter and $\Delta m_{d}$ is less than $10 \%$. The $\Delta z$ distributions for mixed and unmixed events and the resulting time-dependent asymmetry are shown in figure 3, together with the fit result. The neutral $B$
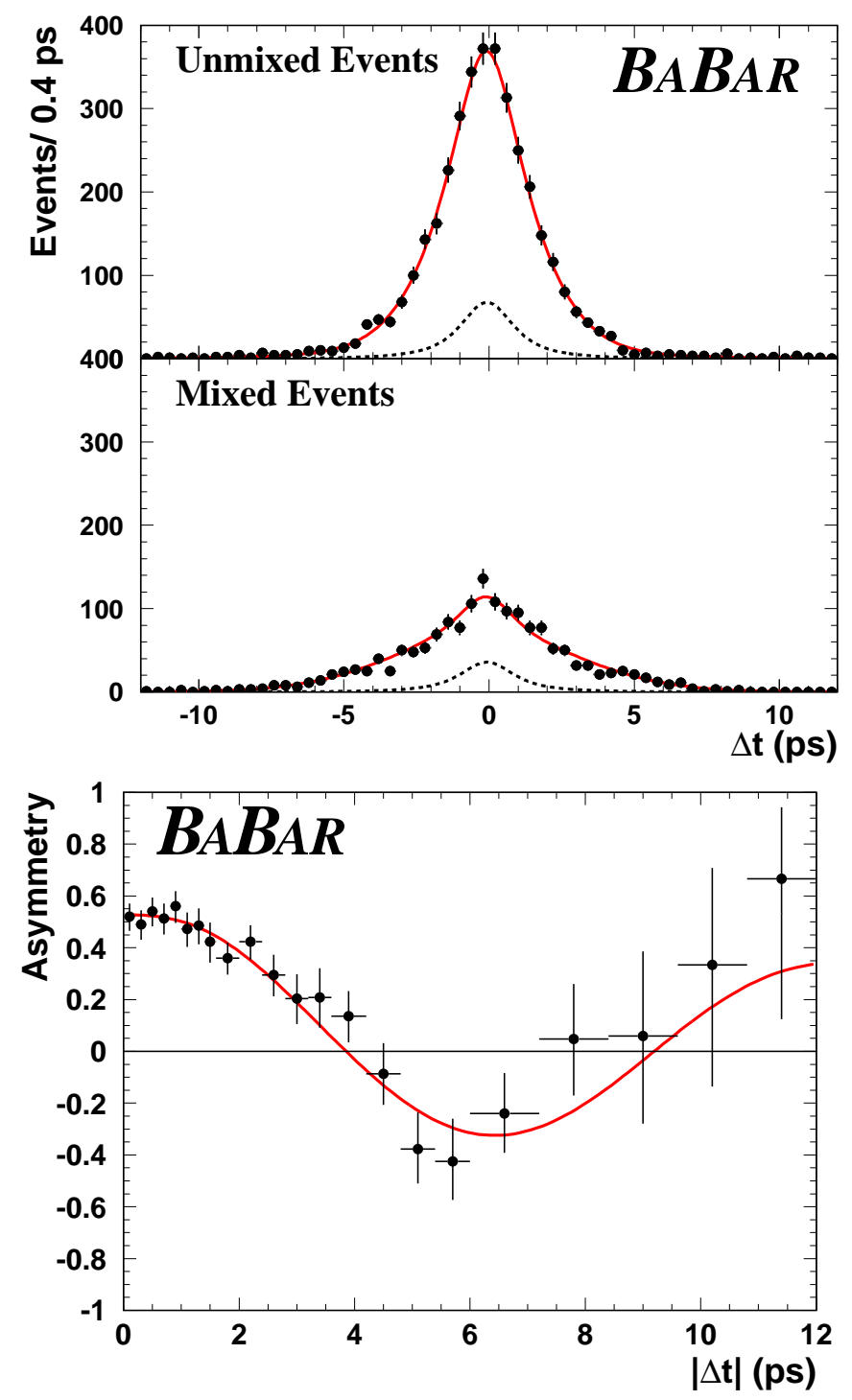

Figure 3: Top: $\Delta t$ distributions for unmixed and mixed neutral $B$ events. Bottom: time dependent asymmetry. The points represent data, the continuous line is the fit result, the dashed line is the background contribution. 
oscillation frequency is measured to be

$$
\Delta m_{d}=\left(0.519 \pm 0.020_{\text {stat }} \pm 0.016_{\text {syst }}\right) \hbar p s^{-1},
$$

and the systematic uncertainty is dominated by resolution and background modeling.

Shortly after this Conference, the charged and neutral $B$ meson lifetime measurements presented in ICHEP2000 [5] were also updated to the full 1999-2000 data sample (figure 国). The new results
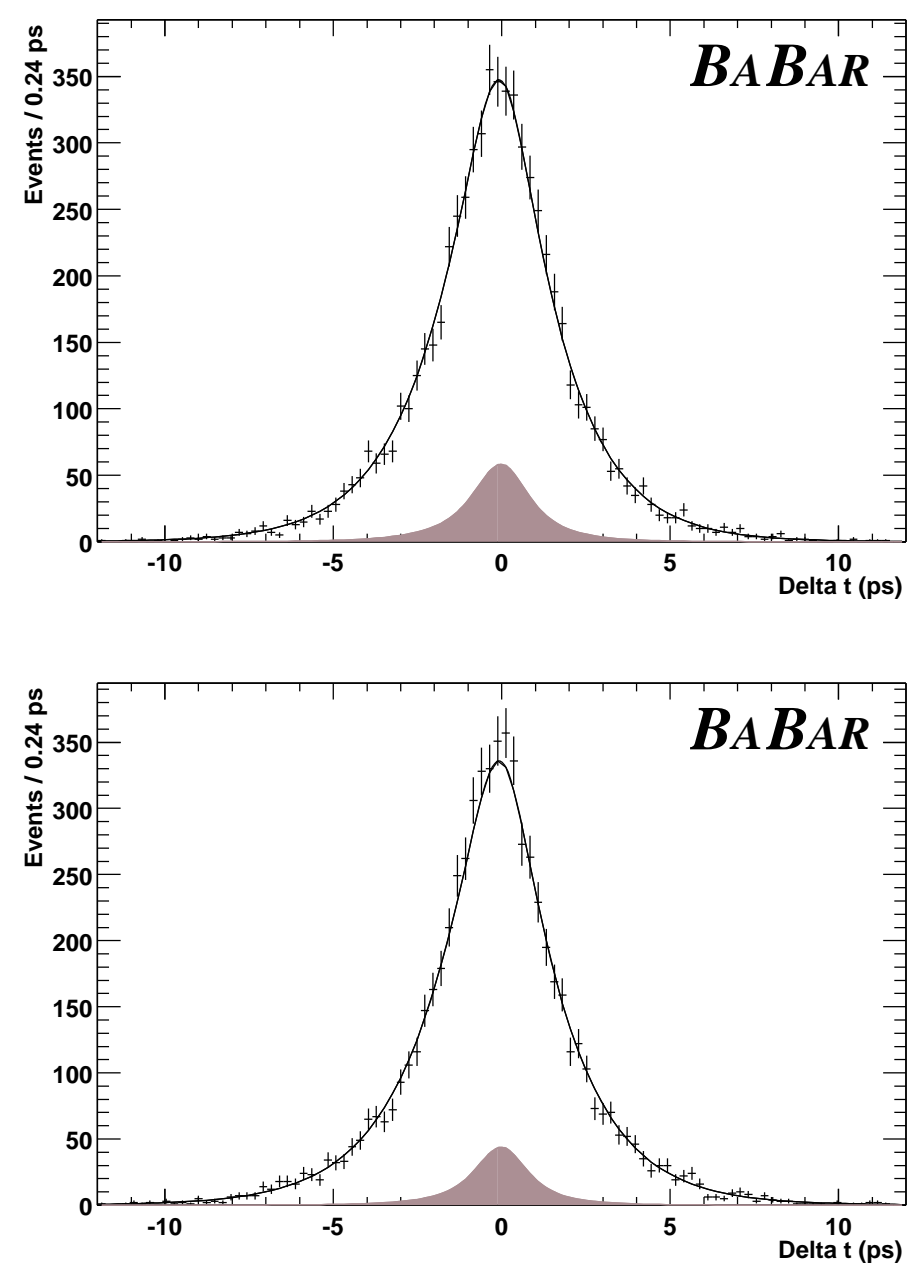

Figure 4: $\Delta t$ distributions for neutral (top) and charged (bottom) fully reconstructed $B$ candidates. The points refer to data, the line is the fit result, the hatched area represents background.

are

$$
\begin{aligned}
\tau_{B^{0}} & =\left(1.546 \pm 0.032_{\text {stat }} \pm 0.022_{\text {syst }}\right) p s, \\
\tau_{B^{+}} & =\left(1.673 \pm 0.032_{\text {stat }} \pm 0.022_{\text {syst }}\right) p s, \\
\tau_{B^{+}} / \tau_{B^{0}} & =1.082_{\text {stat }} \pm 0.026 \pm 0.011_{\text {syst }},
\end{aligned}
$$

in good agreement with world averages. 


\section{$3 \quad$ Mixing with dilepton events}

Measuring $B$ meson properties relying only on two leptons from double semileptonic $B$ decays is conceptually simpler than a fully exclusive analysis, and more powerful in statistical terms, due to the relatively large semileptonic branching fractions and high lepton identification efficiencies. Moreover, the $B$ flavour is easily identified by the charge of the lepton. The asymmetry between unlike- and like-sign dilepton events evolves as

$$
\frac{N_{\ell^{+} \ell^{-}}(\Delta t)-N_{\ell^{+} \ell^{+}, \ell^{-} \ell^{-}}(\Delta t)}{N_{\ell^{+} \ell^{-}}(\Delta t)+N_{\ell^{+} \ell^{+}, \ell^{-} \ell^{-}}(\Delta t)}=\cos \left(\Delta m_{d} \Delta t\right) .
$$

However, the main drawbacks of this approach are the presence of non negligible backgrounds due to leptons from the $b \rightarrow c \rightarrow \ell$ decay chain (cascade decays), which are also the main source of wrong tags, and most seriously, the presence of charged and neutral $B$ mesons in almost equal proportion in the selected sample, which dilutes the mixing asymmetry.

Background from cascade decays is minimized with a neural network technique which uses five input variables (the lepton momenta and opening angle, and the total and missing energies, all in the CMS), and offers better performance in terms of both signal efficiency and mistag rate with respect to a traditional approach based on high momentum cuts.

The fraction $R$ of charged $B$ in the selected sample can be either fit in data or taken (with further assumptions) from previous measurements at the $\Upsilon(4 \mathrm{~S})$. In the former case, the statistical accuracy on the mixing measurement decreases substantially, due to the high correlation between the oscillation frequency $\Delta m_{d}$ and $R$. In the latter case the systematic uncertainty is dominated by the knowledge of $R$. With the current data sample either approach gives similar total uncertainties. However, the present analysis fits $R$ because in the fitting procedure this parameter absorbs any difference in efficiency or mistag rate between charged and neutral $B$ decays, which would be otherwise another source of systematic uncertainty.

Other selection criteria of the dilepton analysis include cuts on event shape variables to suppress continuum background, on track quality to improve the $\Delta z$ resolution function, and invariant mass cuts to reject backgrounds from $J / \psi$ decays and photon conversions. The signal purity after all cuts is $78 \%$. The main backgrounds are due to events with at least one lepton from cascade decays $(12 \%)$, to events with at least one fake lepton $(5 \%)$, and to continuum events $(5 \%)$.

The $z$ difference between $B$ decay vertices is defined by the $z$ difference of the point of closest approach of the leptons to a $\Upsilon(4 \mathrm{~S})$ vertex in the transverse plane. This vertex is determined with a chi-square fit which uses the two lepton tracks and a beam spot constraint. The resulting $\Delta z$ resolution, estimated from signal Monte-Carlo events, has core and tail widths of about $90 \mu \mathrm{m}$ and $200 \mu \mathrm{m}$ respectively, and $75 \%$ of the events in the core. A comparison of the $\Delta z$ distribution for leptons from inclusive $J / \psi$ decays shows an agreement within $10 \%$ between data and simulation.

The analysis presented in ICHEP2000 [6] has been updated shortly after this Conference by using the entire 1999-2000 data sample. About 100000 events are selected. In the analysis update, the total dilepton sample is divided in two independent subsamples, enriched respectively in neutral and charged $B$ decays. This is accomplished by reconstructing inclusively the $\bar{B}^{0} \rightarrow D^{*+} \ell^{-} \nu$ decay, where the $D^{*}$ properties are inferred from the soft pion produced in its decay [7]. Pions of less than $190 \mathrm{MeV} / c$ momentum are identified, the $D^{*}$ energy and momentum are computed and the squared missing mass of the neutrino $M_{\nu}^{2}$ is computed for both lepton/soft pion combinations. An event is assigned to the $B^{0}$-enriched sample if a combination satisfies $\left|M_{\nu}^{2}\right| \leq 1.5\left(\mathrm{GeV} / \mathrm{c}^{2}\right)^{2}$, 
to the $B^{+}$-enriched sample otherwise $e^{5}$. Both subsamples provide similar statistical power to the $\Delta m_{d}$ measurement. A simultaneous binned maximum likelihood fit of the same- and opposite-sign leptons time distributions on both subsamples gives

$$
\Delta m_{d}=\left(0.499 \pm 0.010_{\text {stat }} \pm 0.012_{\text {syst }}\right) \hbar p s^{-1},
$$

which is at present the most precise single measurement of the neutral $B$ meson oscillation frequency. Figure 5 shows the time-dependent asymmetries for the two subsamples, together with the fit results. The systematic uncertainties are dominated by the knowledge of the resolution function

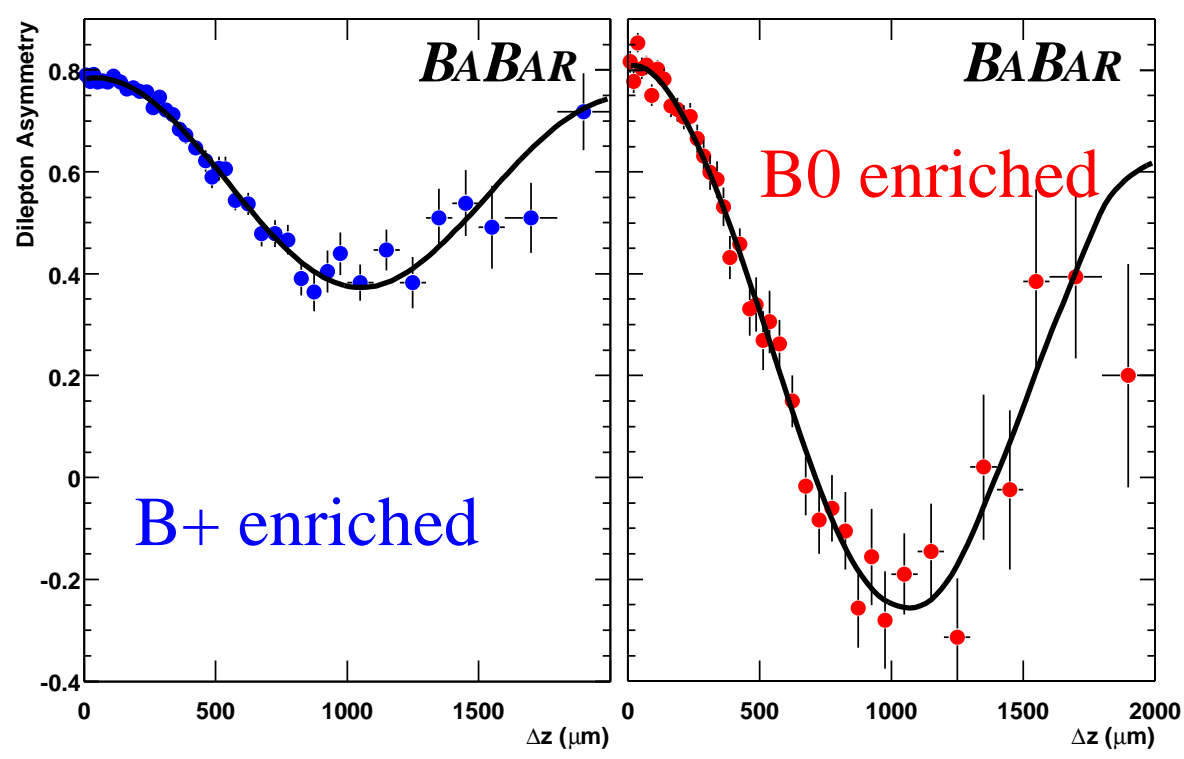

Figure 5: $\Delta t$ asymmetry between opposite and same sign dilepton events, for the $B^{+}$-enriched (left) and $B^{0}$-enriched (right) samples. The points represent data, the line is the fit result.

(0.009), the background parametrization (0.006) and the error on the $B$ meson lifetimes (0.004), which are fixed to the PDG2000 [8] values. Separate fits to the two independent subsamples give $\Delta m_{d}=\left(0.498 \pm 0.015_{\text {stat }}\right) \hbar p s^{-1}$ and $\Delta m_{d}=\left(0.504 \pm 0.014_{\text {stat }}\right) \hbar p s^{-1}$ for the $B^{+}$-enriched and $B^{0}$ enriched samples, respectively. These results are consistent with an independent analysis, based on an unbinned maximum likelihood fitting technique, with the same or even with a different, momentum-cut based, event selection.

\section{Overview of other lifetimes and mixing results}

The following results were presented at ICHEP2000 [9, 10] and are being updated with the full 1999-2000 BABAR data sample.

\footnotetext{
${ }^{3}$ if both combinations satisfy the cut, the one with the smallest $\left|M_{\nu}^{2}\right|$ is chosen.
} 


\subsection{Mixing from fully reconstructed $B^{0} \rightarrow D^{*} \ell \nu$ decays}

This measurement is very similar to the mixing measurement with fully reconstructed hadronic decays, the only difference being in the flavour eigenstate, namely $B^{0} \rightarrow D^{*} \ell \nu$ with $D^{*} \rightarrow D^{0} \pi^{+}$. Neutral $D$ mesons are reconstructed in the $K^{-} \pi^{+}, K^{-} \pi^{+} \pi^{-} \pi^{+}$and $K^{-} \pi^{+} \pi^{0}$ decay modes. By using a data sample corresponding to a luminosity of $8.9 \mathrm{fb}^{-1}$ at the $\Upsilon(4 \mathrm{~S})$ resonance, $7517 \pm 104$ $B^{0} \rightarrow D^{*} \ell \nu$ are reconstructed and the mixing parameter is measured to be

$$
\Delta m_{d}=\left(0.508 \pm 0.020_{\text {stat }} \pm 0.022_{\text {syst }}\right) \hbar p s^{-1} .
$$

\subsection{Lifetimes from partial reconstruction techniques}

Partial reconstruction techniques allow a dramatic enhancement in statistical power, but they are more sensitive to the parametrization of physics processes and to biases in the vertex reconstruction, the latter due essentially to track swappings between the two $B$ mesons. Two decay chains are partially reconstructed:

- $B^{0} \rightarrow D^{*} \pi$, where the missing mass due to the non-reconstructed $D^{0}$ meson is computed by using only the (fast) pion from the $B$ decay, the (slow) pion from the $D^{*}$ decay and the appropriate kinematic constraints. About 1700 events are selected by requiring $M_{\text {miss }}>1.854$ $\mathrm{GeV} / \mathrm{c}^{2}$ in $7.4 \mathrm{fb}^{-1} \Upsilon(4 \mathrm{~S})$ data.

- $B^{0} \rightarrow D^{*} \ell \nu$, where the same slow pion technique already described in Section 3 is applied. The signature of a signal event is a pair of oppositely charged lepton-soft pion tracks, whereas same sign pairs are used to determine the amount (about $40 \%$ of the entire sample) and time dependence of the combinatorial background. The analysis selects about 90000 signal events on $7.4 \mathrm{fb}^{-1} \Upsilon(4 \mathrm{~S})$ data.

The neutral $B$ lifetime is measured to be

$$
\begin{aligned}
& \tau_{B^{0}}=\left(1.55 \pm 0.05_{\text {stat }} \pm 0.07_{\text {syst }}\right) p s \quad\left(B^{0} \rightarrow D^{*} \pi\right), \\
& \tau_{B^{0}}=\left(1.63 \pm 0.02_{\text {stat }} \pm 0.09_{\text {syst }}\right) p s \quad\left(B^{0} \rightarrow D^{*} \ell \nu\right) .
\end{aligned}
$$

\section{Conclusion}

Asymmetric B-Factories offer the unique possibility of precision lifetimes and mixing measurements in the $B$ meson sector. Any single measurement performed with the presently available data is comparable to the corresponding world average. The BABAR experiment at PEP-II has investigated different reconstruction techniques, ranging from the full reconstruction of a $B$ meson decay chain to the totally inclusive selection of events with two leptons from semileptonic decays, to the partial reconstruction of hadronic and semileptonic decays. Recent preliminary measurements of the charged and neutral lifetimes and oscillation frequency with exclusive hadronic decays give

$$
\begin{aligned}
\tau_{B^{0}} & =\left(1.546 \pm 0.032_{\text {stat }} \pm 0.022_{\text {syst }}\right) p s \\
\tau_{B^{+}} & =\left(1.673 \pm 0.032_{\text {stat }} \pm 0.022_{\text {syst }}\right) p s \\
\tau_{B^{+}} / \tau_{B^{0}} & =1.082 \pm 0.026_{\text {stat }} \pm 0.011_{\text {syst }}, \\
\Delta m_{d} & =\left(0.519 \pm 0.020_{\text {stat }} \pm 0.016_{\text {syst }}\right) \hbar p s^{-1} .
\end{aligned}
$$


A preliminary measurement of the mixing parameter with inclusive dilepton events gives

$$
\Delta m_{d}=\left(0.499 \pm 0.010_{\text {stat }} \pm 0.012_{\text {syst }}\right) \hbar p s^{-1},
$$

which is at present the most precise single measurement of this quantity.

Further results on lifetimes and mixing with other techniques are being released in a short time scale.

\section{Acknowledgments}

I wish to thank my BABAR colleagues Riccardo Faccini, David Kirkby, Sören Prell, Gerhard Raven, Jan Stark, Vivek Sharma and Christophe Yèche for useful discussions.

\section{References}

\section{References}

[1] BABAR Collaboration, hep-ex/0012042 (submitted to ICHEP2000, Osaka, Japan).

[2] A. J. S. Smith, this Conference.

[3] BABAR Collaboration, hep-ex/0102030 (accepted for publication on Phys. Rev. Lett.).

[4] Argus Collaboration, Z. Phys. C 48, 543 (1990).

[5] BABAR Collaboration, hep-ex/0008060 (submitted to ICHEP2000, Osaka, Japan).

[6] BABAR Collaboration, hep-ex/0008054 (submitted to ICHEP2000, Osaka, Japan).

[7] CLEO Collaboration, Phys. Rev. Lett. 71, 1680 (1993).

[8] Particle Data Group, Eur. Phys. J. C 15, 1 (2000).

[9] BABAR Collaboration, hep-ex/0008052 (submitted to ICHEP2000, Osaka, Japan).

[10] BABAR Collaboration, hep-ex/0008053 (submitted to ICHEP2000, Osaka, Japan). 